\title{
The threshold of bone mineral density for vertebral fractures in female patients with primary hyperparathyroidism
}

\author{
Hiroshi Kaji, Mika Yamauchi ${ }^{1}$, Kazuo Chihara and Toshitsugu Sugimoto ${ }^{1}$ \\ Division of Endocrinology/Metabolism, Neurology and Hematology/Oncology, Department of Clinical Molecular Medicine, Kobe University Graduate \\ School of Medicine, 7-5-2 Kusunoki-cho, Chuo-ku, Kobe 650-0017, Japan and ${ }^{1}$ Department of Endocrinology, Metabolism and Hematological Oncology, \\ Shimane University School of Medicine, 89-1, Enya-cho, Izumo, Shimane 693-8501, Japan
}

(Correspondence should be addressed to H Kaji; Email: hiroshik@med.kobe-u.ac.jp)

\begin{abstract}
Background and objective: Primary hyperparathyroidism (pHPT) is one of the causal diseases that induce secondary osteoporosis. Although patients with pHPT have reduced bone mineral density (BMD) especially at the cortical bone, there have been controversies about risk of fracture. Moreover, no reports have been available about the threshold of BMD for fractures in pHPT patients.

Methods: BMD values were measured by dual-energy x-ray absorptiometry at lumbar spine, femoral neck and distal one third of radius. Various indices were compared in 116 female pHPT patients and 716 control subjects. Moreover, we analyzed relationship between the cut-off values of BMD and the prevalence of vertebral fractures in pHPT and control subjects.

Results: The prevalence of subjects with vertebral fractures was lower in pHPT patients, compared with that of control subjects. Age and body height were significantly higher and lower in pHPT women with vertebral fractures, respectively. Lumbar spine BMD was significantly lower in pHPT women with vertebral fractures, presumably due to their increased age. There were no differences in femoral neck and radius BMD or in bone metabolic indices between pHPT women with and without vertebral fractures. On the other hand, age-matched BMD was not significantly different between both groups at any measured site. Cut-off values of BMD at lumbar spine and femoral neck were lower in postmenopausal $\mathrm{pHPT}$ patients, compared with those of the postmenopausal control group. Moreover, cut-off values of BMD at radius was much lower in postmenopausal pHPT patients, compared with those of the postmenopausal control group (pHPT vs control $\left(\mathrm{g} / \mathrm{cm}^{2}\right): 0.670 \mathrm{vs} 0.706$ at lumbar spine; 0.549 vs 0.570 at femoral; 0.394 vs 0.474 at radius). Sensitivity and specificity of vertebral fractures was lower in pHPT patients, compared with those in control group.

Conclusions: The present cross-sectional study demonstrated that thresholds of BMD for vertebral fractures were lower especially at radial bone in female patients with pHPT, compared with those in the control group.
\end{abstract}

European Journal of Endocrinology 153 373-378

\section{Introduction}

Primary hyperparathyroidism (pHPT) is recognized as a disorder that frequently causes secondary osteoporosis. In pHPT there is an excess of endogenous parathyroid hormone (PTH) from abnormally functioning parathyroid glands. Although the intermittent administration of low dose PTH increases bone mass in animals and humans (1), patients with pHPT have reduced bone mineral density (BMD), especially at the cortical bone $(1,2)$. Our recent study revealed that both cortical and trabecular bone mass were reduced in pHPT patients by volumetric BMD analysis with peripheral quantitative computed tomography (pQCT) in female subjects (3). The findings of this study also suggest an excess of endogenous PTH-stimulated periosteal bone formation in pHPT patients, which might partly compensate for the decrease in bone strength induced by low BMD (3). Moreover, several studies suggest that bone structure may be improved in pHPT, although the effects of pHPT on bone structure seem to change in a manner dependent on the severity of disease or the type of bone (4-7). These findings raised the possibility that bone might be resistant to fractures, in spite of reduced BMD, in pHPT patients.

There have been controversies about fracture risk in pHPT patients. Khosla et al. reported that pHPT was associated with an increased risk of vertebral, forearm, rib and pelvic fractures (8). In that study, age and female gender were independent predictors of fracture risk. Moreover, other groups reported the increased risk of vertebral and forearm fractures in pHPT patients 
and subsequent decrease in fracture risk after parathyroidectomy $(9-12)$. However, several studies did not find increased risk of fractures and reduced fracture risk after operation $(13,14)$. Thus, controversies still exist about the relationship between the decrease in BMD and fracture risk in patients with pHPT. Moreover, no reports have been available about the threshold of BMD for fractures in pHPT patients.

The present study was, therefore, performed to examine the relationship between vertebral fractures and various indices including BMD in 116 female pHPT patients. Moreover, we analyzed the relationship between cut-off values of BMD and the prevalence of vertebral fractures in patients with pHPT and compared these values with those of control subjects.

\section{Subjects and methods}

\section{Subjects}

One hundred and sixteen female patients who were diagnosed as pHPT and 716 control subjects participated in this study. Control subjects were Japanese women who visited our outpatient clinic to see whether they might be suffering from osteoporosis. None of the control subjects had hepatic or renal dysfunction, thyroid disease or systemic diseases, which might affect bone metabolism. All subjects were free of drugs known to influence bone metabolism up to the time of the present study. Postmenopausal subjects were 100 and 622 in pHPT and control groups, respectively. In all pHPT patients enrolled in the present study, abnormal parathyroid gland swelling was successfully identified by at least two imaging techniques among ultrasonography, computed tomography, magnetic resonance imaging or technetium sestamibi scintigraphy; and biochemical data were compatible with pHPT. Patients whose diagnosis was ambiguous were excluded from the study, for example, cases in which serum calcium was high and serum PTH levels were in the upper normal range. Moreover, familial hypocalciuric hypercalcemia was excluded, based on low calcium:creatinine clearance ratio. pHPT patients taking drugs or with other systemic diseases, affecting bone metabolism, were excluded from the study. The numbers of fractured patients with pHPT were seven, five and two for one, two and three vertebral fractures, respectively. The patients with pHPT were not separated according to severity of disease. The study was crosssectional and approved by the ethical review board of Kobe University Hospital. All subjects agreed to participate in the study and gave informed consent.

\section{Biochemical measurements}

Serum and urinary chemistry determinations were performed by standard automated techniques. Serum chemistry was performed in the daily routine assays.
Urine was collected as second void morning urine. Serum concentrations of intact PTH were measured by immunoradiometric assay (Allegro Intact PTH IRMA kit, Nichols Institute Diagnostics, San Juan Capistrano, CA, USA; normal range, $10-65 \mathrm{ng} / \mathrm{l}$ ), as previously described (3).

Serum levels of osteocalcin and urinary levels of deoxypyridinoline (Dpd) were measured as previously described (15).

\section{Radiography}

Lateral radiographs of the thoracic and lumbar spine were taken. The anterior, central and posterior heights of each of the 13 vertebral bodies from $\mathrm{T}_{4}$ to $\mathrm{L}_{4}$ were measured using an electronic caliper. Vertebral fractures were diagnosed to be present if at least one of three height measurements taken from along the length of the same vertebra was decreased by more than $20 \%$ compared with the height of the nearest uncompressed vertebral body. Defining vertebral fractures from radiographs of the spine is difficult, because there is no gold standard for what types of deformities of vertebral shape are the results of breakage of bone. Definitions of vertebral fractures with high true positive rates and low false positive rates are clinically useful in identifying women who may have vertebral fractures. The criterion in the present study $(>20 \%)$ was considered to be good for defining vertebral fractures because it had a relatively high true positive rate and low false positive rate based on qualitative classifications from a previous report (16).

\section{BMD measurements by DXA}

BMD values were measured by dual-energy x-ray absorptiometry (DXA) using QDR-2000 (Hologic Inc., Waltham, MA, USA) at lumbar spine $\left(\mathrm{L}_{2-4}\right)$, femoral neck (FN) and distal one third of radius (Rad 1/3). The vertebrae with vertebral fractures or overt osteoarthrosis were excluded from the analysis of BMD, because these factors may increase BMD through artifacts. BMD was automatically calculated from the bone area $\left(\mathrm{cm}^{2}\right)$ and bone mineral content (BMC; g) and expressed absolutely in $\mathrm{g} / \mathrm{cm}^{2}$. The Z-score is the number of S.D. a given measurement differs from the mean for a sex-, age-, and race-matched reference population. The T-score is the number of S.D. a given measurement differs from the mean for a normal young adult reference population. The coefficients of variation (precision) of measurements of the lumbar spine, femoral neck and radius were 0.9, 1.7 and $1.9 \%$, respectively.

\section{Statistical analysis}

All data were expressed as the mean \pm S.D. for each index. Comparisons of each group were made with 
the non-parametric Mann-Whitney U-test. $P$ values $<0.05$ were considered significant. To compare the strength of association between BMD at each of the measurement sites and fractures, we analyzed the areas under receiver operating characteristic (ROC) curves for each site (17). For each of the BMD measurements at the radius, femoral and lumbar spine sites and for each of the vertebral fracture groups, all possible cutoff points were defined and the proportion of subjects without fractures above each point (the specificity) and the proportion of subjects with fractures below each point (the sensitivity) were calculated. This yields ROC curve that displays the relationship between sensitivity and specificity for each BMD measurement as a discriminator between the normal and fracture groups. The optimal criterion, which attempts to maximize both sensitivity and specificity, is usually defined as the point closest to $100 \%$ sensitivity and $100 \%$ specificity.

On the curve, the point that is closest to the upper left corner of the graph is judged as the optimal criterion.

\section{Results}

\section{Background data}

Baseline indices are shown in Tables 1 and 2 in pHPT and control groups. Fourteen and 158 women were diagnosed as having one or more vertebral fractures in pHPT and control groups, respectively. The prevalence of subjects with vertebral fractures was lower in pHPT patients, compared with that of control subjects. The differences in the prevalence of vertebral fractures between control subjects and pHPT patients were significant $\left(\chi^{2}=2.345 ; P=0.0132\right)$. Body weight and body mass index (BMI) were significantly higher in pHPT patients, compared with the control group. $\mathrm{BMD}$ as well as Z-score (age-matched BMD) was lower in pHPT patients at lumbar spine, femoral neck and radius. The extent of reduced BMD was marked in radius, and lumbar BMD was relatively preserved. BMD at any site was significantly related to age and body weight when a multiple regression analysis was performed with BMD as the dependent variable, and age and weight as independent variables (data not shown).

As shown in Table 2, serum levels of calcium and intact PTH were elevated in pHPT patients. Moreover, serum levels of non-specific alkaline phosphatase and osteocalcin as well as urinary Dpd were higher in pHPT patients, indicating that bone turnover was increased in pHPT patients.

\section{Comparison of various indices between pHPT patients with and without vertebral fractures}

We compared various indices between pHPT patients, with and without vertebral fractures, when the diagnosis of pHPT was performed. As shown in Table 3, age and body height were significantly higher and lower, respectively, in the group with fracture. Body weight and BMI were similar in both groups. Serum levels of calcium, phosphorus and ALP were 2.95 \pm 0.31 $\mathrm{mmol} / \mathrm{l}$ vs $2.80 \pm 0.19 \mathrm{mmol} / \mathrm{l}, \quad 0.85 \pm 0.18 \mathrm{mmol} / \mathrm{l}$ vs $0.84 \pm 0.14 \mathrm{mmol} / \mathrm{l}$ and $467.8 \pm 483.8 \mathrm{IU} / \mathrm{l}$ vs $435.6 \pm 229.3 \mathrm{IU} / \mathrm{l}$ in the groups without and with vertebral fractures, respectively (no significant difference). Lumbar spine BMD was significantly lower in women with fractures. There were no differences in femoral neck and radius BMD between pHPT women with and without vertebral fractures. On the other hand, age-matched BMD (Z-scores) were not significantly different in both groups at any measured site, suggesting that reduced lumbar spine BMD in women with fractures was largely due to their increased age.

Table 1 Background data in control and pHPT groups.

\begin{tabular}{lcr}
\hline & Control & pHPT \\
\hline Number of subjects & 716 & 116 \\
Number of subjects with vertebral fractures & $158(22.1 \%)$ & $14(12.1 \%)^{\star *}$ \\
Number of postmenopausal women & $622(86.9 \%)$ & $100(86.2 \%)$ \\
Age (years) & $61.2 \pm 10.9$ & $60.0 \pm 13.1$ \\
Body height $(\mathrm{cm})$ & $152.5 \pm 5.8$ & $151.9 \pm 6.2$ \\
Body weight $(\mathrm{kg})$ & $51.0 \pm 7.9$ & $53.1 \pm 8.8^{\star}$ \\
BMl $\left(\mathrm{kg} / \mathrm{m}^{2}\right)$ & $21.9 \pm 3.0$ & $23.0 \pm 3.9^{\star *}$ \\
L2-4BMD $\left(\mathrm{g} / \mathrm{cm}^{2}\right)$ & $0.781 \pm 0.157$ & $0.711 \pm 0.154^{\star *}$ \\
T-score & $-2.324 \pm 1.502$ & $-2.785 \pm 1.539^{\star *}$ \\
Z-score & $-0.028 \pm 1.285$ & $-0.704 \pm 1.256^{\star *}$ \\
FN BMD $\left(\mathrm{g} / \mathrm{cm}^{2}\right)$ & $0.617 \pm 0.110$ & $0.567 \pm 0.094^{\star *}$ \\
T-score & $-1.573 \pm 1.048$ & $-2.052 \pm 0.897^{\star *}$ \\
Z-score & $0.096 \pm 1.307$ & $-0.640 \pm 1.065^{\star *}$ \\
Rad1/3 BMD $\left(\mathrm{g} / \mathrm{cm}^{2}\right)$ & $0.508 \pm 0.086$ & $0.438 \pm 0.096^{\star *}$ \\
T-score & $-3.024 \pm 1.680$ & $-4.389 \pm 1.874^{\star *}$ \\
Z-score & $-0.464 \pm 1.400$ & $-1.908 \pm 1.910^{\star *}$ \\
\hline
\end{tabular}

Control vs pHPT: *, $P<0.01 ; * \star, P<0.05$ 
Table 2 Background data in pHPT groups.

\begin{tabular}{lcc}
\hline & Normal range & pHPT \\
\hline Serum calcium $(\mathrm{mmol} / \mathrm{l})$ & $2.10-2.48$ & $2.95 \pm 0.32(2.33-4.03)$ \\
Serum creatinine $(\mathrm{mg} / \mathrm{l})$ & $0.05-0.13$ & $0.070 \pm 0.026(0.04-0.2)$ \\
ALP $(\mathrm{IU} / \mathrm{l})$ & $100-303$ & $464.0 \pm 460.9(125-4027)$ \\
Intact PTH $(\mathrm{ng} / \mathrm{l})$ & $10-65$ & $207.2 \pm 238.4(38-1400)$ \\
Osteocalcin $(\mu \mathrm{g} / \mathrm{l})$ & $2.5-13$ & $22.18 \pm 20.43(2-100)$ \\
u-Ca/u-Cr & $<0.3$ & $0.42 \pm 0.17(0.043-0.85)$ \\
u-Dpd $(\mathrm{pmol} / \mu \mathrm{mol} . \mathrm{Cr})$ & $2.8-7.6$ & $18.46 \pm 15.74(1-66.5)$ \\
\hline
\end{tabular}

\section{Cut-off values for vertebral fractures}

Table 4 shows cut-off values of BMD for vertebral fractures at the point of coincidence between sensitivity and specificity calculated by ROC analyses. The cut-off value discriminates the patients with vertebral fractures from those without fractures. Cut-off values of BMD at lumbar spine and femoral neck were lower in postmenopausal pHPT patients, compared with those of the postmenopausal control group. Moreover, cutoff values of BMD at radius was much lower in postmenopausal pHPT patients, compared with those of the postmenopausal control group. Sensitivity and specificity were lower in pHPT patients, compared with those in control group.

\section{Discussion}

Body weight and BMI were significantly higher in pHPT patients, compared with those in control group. These data were compatible with previous reports suggesting increased BMI in pHPT patients (18). BMD as well as Z-score was lower in pHPT patients at lumbar spine, femoral neck and radius, which were compatible with reduced BMD in pHPT patients (1-3). In the present study, we compared various indices between women with and without vertebral fractures in pHPT patients. Age was significantly higher in the group with fractures, which might explain the lower lumbar spine BMD in patients with fractures, as age-matched BMD was not different between both groups. Previous reports indicate that age is an independent predictor of fracture risk, and that a combination of age and BMD is most important in determining fracture risk (8), which supports the present evidence. Lower height in the group with vertebral fractures is considered to be partly due to vertebral deformity by the fractures themselves, leading to significant loss in body height. As for BMD, the differences in agematched BMD was less than those of the absolute values. Only lumbar spine BMD was lower in the fracture group, but radial and femoral BMD were not different between both groups. These data suggest that BMD measurement, which is specific to the fracture sites, is important in the prediction of fracture risk. Although reduced BMD in the forearm is common in pHPT patients, reduced radial BMD is not considered to be a risk factor in the prediction of vertebral fracture risk.

The elevation of bone metabolic indices is related to the severity of pHPT, and our previous study indicated that the level of serum alkaline phosphatase was the predictor of bone mass change after parathyroidectomy in pHPT patients (2). Although the relationship between fracture risk and bone metabolic indices in pHPT is still unknown, serum levels of ALP and osteocalcin, as well as urinary Dpd, were not significantly different between pHPT patients with and without vertebral fractures in the present study. These data suggest that disease severity of pHPT is not related to the risk of vertebral fractures.

Table 3 Comparison of various indices between women with and without vertebral fractures in pHPT group.

\begin{tabular}{lcc}
\hline & & Vertebral fractures \\
\cline { 2 - 3 } pHPT & $(-)$ & $(+)$ \\
\hline Number of subjects & 102 & 14 \\
Age (years) & $58.7 \pm 13.0$ & $69.6 \pm 9.3^{\star}$ \\
Body height $(\mathrm{cm})$ & $152.4 \pm 5.8$ & $147.5 \pm 7.1^{*}$ \\
Body weight $(\mathrm{kg})$ & $53.2 \pm 8.5$ & $51.1 \pm 11.5$ \\
BMl $\left(\mathrm{kg} / \mathrm{m}^{2}\right)$ & $22.9 \pm 3.7$ & $23.5 \pm 4.8$ \\
L2-4BMD $\left(\mathrm{g} / \mathrm{cm}^{2}\right)$ & $0.724 \pm 0.149$ & $0.612 \pm 0.166^{\star *}$ \\
T-score & $-2.665 \pm 1.490$ & $-3.624 \pm 1.678^{\star *}$ \\
Z-score & $-0.692 \pm 1.211$ & $-0.798 \pm 1.619$ \\
FN BMD $\left(\mathrm{g} / \mathrm{cm}^{2}\right)$ & $0.574 \pm 0.095$ & $0.530 \pm 0.086$ \\
T-score & $-0.692 \pm 1.211$ & $-2.398 \pm 0.824$ \\
Z-score & $-0.634 \pm 1.060$ & $-0.667 \pm 1.144$ \\
Rad1/3 BMD $\left(\mathrm{g} / \mathrm{cm}^{2}\right)$ & $0.441 \pm 0.097$ & $0.414 \pm 0.087$ \\
T-score & $-4.337 \pm 1.893$ & $-4.856 \pm 1.699$ \\
Z-score & $-1.981 \pm 1.900$ & $-1.262 \pm 1.969$ \\
Intact PTH $(\mathrm{ng} / \mathrm{l})$ & $215.9 \pm 251.0(38-1400)$ & $133.1 \pm 103.8(46-401)$ \\
Osteocalcin $(\mu \mathrm{g} / \mathrm{l})$ & $23.7 \pm 21.5$ & $13.7 \pm 9.32$ \\
u-Dpd $(\mathrm{pmol} / \mu \mathrm{mol} . \mathrm{Cr})$ & $18.3 \pm 15.6$ & $19.1 \pm 19.7$ \\
\hline
\end{tabular}

*, $P<0.01 ; * *, P<0.05$ 
Table 4 Cut-off values for vertebral fractures at the point of coincidence between sensitivity and specificity in postmenopausal women.

\begin{tabular}{|c|c|c|c|c|c|}
\hline & \multicolumn{3}{|c|}{ Cut-off value } & \multirow{3}{*}{$\begin{array}{c}\text { Sensitivity } \\
(\%)\end{array}$} & \multirow{3}{*}{$\begin{array}{c}\text { Specificity } \\
(\%)\end{array}$} \\
\hline & \multirow[b]{2}{*}{$\left(\mathrm{g} / \mathrm{cm}^{2}\right)$} & \multicolumn{2}{|c|}{ T-score } & & \\
\hline & & & $(\%)$ & & \\
\hline \multicolumn{6}{|l|}{ Control } \\
\hline L2-4 & 0.706 & -2.77 & 70 & 73.0 & 73.0 \\
\hline Femoral neck & 0.570 & -2.02 & 72 & 70.8 & 70.8 \\
\hline $\begin{array}{l}\text { Radius 1/3 } \\
\text { pHPT }\end{array}$ & 0.474 & -3.69 & 72 & 70.9 & 70.9 \\
\hline L2-4 & 0.670 & -3.10 & 66 & 59.6 & 59.6 \\
\hline Femoral neck & 0.549 & -2.22 & 69 & 62.0 & 62.0 \\
\hline Radius $1 / 3$ & 0.394 & -5.25 & 59 & 59.9 & 59.9 \\
\hline
\end{tabular}

One hundred pHPT patients and 622 control subjects were included in the analysis.

There have been no reports about the threshold of BMD for fractures in pHPT patients. Present data showed that the thresholds of lumbar spine and femoral neck BMD for vertebral fractures seemed to be lower in pHPT patients, compared with those in the control group. Moreover, the threshold of radial BMD for vertebral fractures was markedly lower in $\mathrm{pHPT}$ patients. These findings support the idea that radial BMD measurement is not a good way to predict the risk of vertebral fractures in pHPT, although radial BMD is useful in predicting osteoporotic fractures (19). In pHPT patients, weight-bearing bones, such as lumbar spine and femoral neck, are relatively preserved compared with radial bone, which represents non-weightbearing cortical bone $(2,20,21)$. However, reduced radial BMD as well as lumbar spine and femoral BMD are factors that contribute to the decision to carry out parathyroidectomy in pHPT patients. The present results suggest that the criteria used to decide whether to carry out surgery be reevaluated. Moreover, the sensitivity and specificity of cut-off values seemed to be lower in pHPT patients, compared with those in the control group, suggesting that BMD is a less reliable marker for the prediction of fracture risk in pHPT, compared with post menopausal osteoporosis. Factors other than BMD might influence bone strength in pHPT patients. Kohsla et al. (8) reported that other peripheral fractures, such as forearm, rib and pelvic fractures, also increased in pHPT patients. Further studies of another fracture group will be necessary.

The reasons for decreased threshold as well as sensitivity and specificity in pHPT patients are still unknown. Bone strength is affected by BMD as well as bone quality, including bone structure, the accumulation of micro damage, bone turnover state, bone matrix protein and calcification. In pHPT patients the indices of trabecular connectivity were similar or higher and the indices of trabecular disconnectivity were reduced compared with controls, despite a high rate of bone turnover (4-7). Moreover, the connectivity of trabecular bone was preserved in pHPT patients in a three-dimensional study with micro computed tomography (22). These data indicate that improved bone structure might antagonize the effects of reduced BMD in pHPT patients, resulting in the decrease in the fracture threshold of BMD. Alternatively, the change in bone geometry might affect bone strength in pHPT patients. Our previous study revealed that total bone area and endosteal and periosteal circumferences were significantly higher in pQCT analysis of female pHPT patients (3). Therefore, an excess of endogenous PTH seemed to stimulate periosteal bone formation, which might partly compensate for a decrease in bone strength induced by low BMD (23). Moreover, increased radial bone size might lead to underestimation of radial BMD by DXA on true BMD in pHPT. In the present data, body weight and BMI were significantly higher in pHPT patients, compared with those in control group. Higher body weight or BMI have a positive impact on bone mass (15). Although the effects of body weight on bone quality are unknown, we cannot rule out the possibility that higher body weight and/or BMI might increase bone strength, resulting in the decreased fracture threshold of BMD.

In the present study, the prevalence of vertebral fractures was lower in the pHPT group compared with those in control group. Since BMD of the control group is relatively lower, probably due to osteopenia or osteoporosis in some subjects, the fracture incidence of the control group might be relatively higher. However, age-matched BMD was actually about 0.6 to 1.5 S.D. lower, depending on site, in the pHPT group compared with that in control group. These findings suggest that the prevalence of vertebral fractures was relatively lower for BMD in postmenopausal pHPT patients. In our data, cut off values for vertebral fractures were relatively lower in pHPT patients. Moreover, age-matched BMD was not significantly lower in the fracture group. Taken together, the bone of pHPT might be resistant to vertebral fractures for the degree of bone loss, resulting in the decreased threshold of BMD for vertebral fractures.

T-score of BMD ( $\mathrm{T}<-2.5$ S.D.) is considered to be a factor that may indicate the need for parathyroidectomy in the current NIH BMD guidelines (24). However, the present studies suggested that the threshold of BMD for vertebral fractures is relatively lower compared with that in control subjects, especially at the forearm. These findings raise the possibility that criteria about BMD might now need to be reconsidered. Further studies will be necessary to clarify these issues. There are several limitations in the present study. Since control subjects in the present study were recruited from the out patient clinic and do not represent the general population, some selection bias might be included in the control subjects. Subjects visiting a clinic to see if 
they have osteoporosis often come because of risk factors. In a population-based study in Japan with 1092 females (aged 45-69), the prevalence of vertebral fractures was $4.7 \%$ overall, and $5.7 \%$ for $60-64$ year olds, and $13.0 \%$ for $65-69$ year olds (25). The control subjects may thus have an increased risk of vertebral fractures, compared with the general population. Secondly, definite diagnosis of pHPT comes from histological findings and the normalization of hypercalcemia after parathyroidectomy. This makes the present study limited because we cannot completely rule out the possibility that the diagnosis of pHPT might not be correct in all subjects. Thirdly, 25-hydroxyvitamin D3 data were not measured for the assessment of vitamin D status in the present study. Thus, differences in vitamin D status might affect the BMD threshold for vertebral fractures. Moreover, the length of disease might affect the fracture risk independently of BMD, although this is usually difficult to determine in pHPT.

In conclusion, the present study demonstrates that women with pHPT sustain vertebral fractures at lower BMDs (particularly radial BMD) than control women. The guidelines for surgery in asymptomatic hyperparathyroidism based on an osteoporotic T-score may now need to be reconsidered.

\section{References}

1 Dempster DW, Cosman F, Parisien M, Shen V \& Lindsay R. Anabolic actions of parathyroid hormone on bone. Endocrine Reviews $199314690-709$.

2 Nakaoka D, Sugimoto T, Kobayashi T, Yamaguchi T, Kobayashi A \& Chihara K. Prediction of bone mass change after parathyroidictomy in patients with primary hyperparathyroidism. Journal of Clinical Encocrinology and Metabolism 2000 85 1901-1907.

3 Chen Q, Kaji H, Iu MF, Nomura R, Sowa H, Yamauchi M, Tsukamoto T, Sugimoto T \& Chihara K. Effects of an excess and a deficiency of endogenous parathyroid hormone on volumetric bone mineral density and bone geometry determined by peripheral quantitative computed tomography in female subjects. Journal of Clinical Endocrinology and Metabolism 200388 2655-2658.

4 Uchiyama T, Tanizawa T, Ito A, Endo N \& Takahashi H. Miscrostructure of the trabecula and cortex of iliac bone in primary hyperparathyroidism patients determined using histomorphometry and node-strut analysis. $1999 \mathbf{1 7} 283-288$.

5 Parisien M, Cosman F, Mellish RWE, Schnitzer M, Nieves J, Silverberg SJ, Shane E, Kimmel D, Recker RR, Bilezikian JP, Lindsay R \& Dempster DW. Bone structure in postmenopausal hyperparathyroid, osteoporotic, and normal women. Journal of Bone Mineral Research 199510 1393-1399.

6 Parisien M, Silverberg SJ, Shane E, de la Cruz L, Lindsay R, Bilezkian JP \& Dempster DW. The histomorphometry of bone in primary hyperparathyroidism: preservation of cancellous bone structure. Journal of Clinical Endocrinology and Metabolism 1990 70 930-938.

7 Parisien M, Mellish RWE, Silverberg SJ, Shane E, Lindsay R, Bilezkian JP \& Dempster DW. Maintenance of cancellous bone connectivity in primary hyperparathyroidism: trabecular strut analysis. Journal of Bone Mineral Research $19927913-919$.

8 Kohsla S, Melton III LJ, Wermers RA, Crowson CS, O'Fallon WM \& Riggs BL. Primary hyperparathyroidism and risk of fracture: a population-based study. Journal of Bone Mineral Research 1999 14 1700-1707.

9 Melton III LJ, Atkinson EJ, O’Fallon WM \& Heath III H. Risk of age-related fractures in patients with primary hyperparathyroidism. Archives of Internal Medicine 1992152 269-273.

10 Kenny AM, MacGillivray DC, Pilbeam CC, Crombie HD \& Raisz LG. Fracture incidence in postmenopausal women with primary hyperparathyroidism. Surgery 1995118 109-114.

11 Vestergaard P, Mollerup CL, Frokar VG, Christiansen P, BlichertToft M \& Mosekilde L. Cohort study of risk of fracture before and after surgery for primary hyperparathyroidism. British Medical Journal $2000321598-602$.

12 Vestergaard P \& Mosekilde L. Cohort study on effects of parathyroid surgery on multiple outcomes in primary hyperparathyroidism. British Medical Journal 2003327 530-534.

13 Wilson RJ, Rao DS, Ellis B, Kleerekoper M \& Parfitt AM. Mild asymptomatic primary hyperparathyroidism is not a risk factor for vertebral fractures. Annals of Internal Medicine $1988 \mathbf{1 0 9}$ 959-962.

14 Vestergaard P \& Mosekilde L. Fractures in patients with primary hyperparathyroidism: nationwide follow-up study of 1201 patients. World Journal of Surgery 2003273 43-49.

15 Nakaoka D, Sugimoto T, Kaji H, Kanzawa M, Yano S, Yamauchi M, Sugishita T \& Chihara K. Determinants of bone mineral density and spinal fracture risk in postmenopausal Japanese women. Osteoporosis International 200112 548-554.

16 Smith-Bindman R, Cummings SR, Steiger P \& Genant HK. A comparison of morphometric definitions of vertebral fractures. Journal of Bone Mineral Research 19916 25-34.

17 Hanley JA \& McNail BJ. The meaning and use of the area under a receiver operating characteristic (ROC) curve. Radiology 1982 $14329-36$

18 Grey AB, Evans MC, Stapleton JP \& Reid IR. Body weight and bone mineral density in postmenopausal women with primary hyperparathyroidism. Annals of Internal Medicine $1994 \mathbf{1 2 1}$ 745-749.

19 Marshall D, Johnell O \& Wedel H. Meta-analysis of how well measures of bone mineral density predict occurrence of osteoporotic fractures. British Medical Journal $1996 \mathbf{3 1 2}$ $1254-1259$.

20 Silverberg SJ, Shane E, De La Cruz L, Dempster DW, Feldman F, Seldin D, Jacobs TP, Siris ES, Cafferty M \& Parisien MV. Skeletal disease in primary hyperparathyroidism. Journal of Bone Mineral Research $19894283-291$.

21 Dempster DW, Parisien M, Silverberg SJ, Liang XG, Schenitzer M, Shen V, Shane E, Kimmel DB, Recker R, Lindsay R \& Bilezikian JP. On the mechanism of cancellous bone preservation in postmenopausal women with mild primary hyperparathyroidism. Journal of Clinical Endocrinology and Metabolism $1999 \mathbf{8 4} 1562-1566$.

22 Dempster DW, Caldwell NJ, Goldstein SA, Parisien M, Silverberg SJ, Shane E, Fitzpatrick LA, Lindsay R \& Bilezikian JP. Three dimensional assessment of trabecular architecture in mild primary hyperparathyroidism (PHPT). Journal of Bone Mineral Research 200217 S166.

23 Parfitt AM. Parathyroid hormone and periosteal bone expansion. Journal of Bone Mineral Research 200217 1741-1743.

24 Bilezikian JP, Potts JT Jr, El-Hajj Fuleihan G, Kleerekoper M, Neer R, Peacock M, Rastad J, Silverberg SJ, Udelsman R \& Wells SA Jr. Summary statement from a workshop on asymptomatic primary hyperparathyroidism: a perspective for the 21 st century. Journal of Bone Mineral Research 200217 2-11.

25 Kitazawa A, Kushida K, Yamazaki K \& Inoue T. Prevalence of vertebral fractures in a population-based sample in Japan. Journal of Bone Mineral Metabolism 200119 115-118.

Received 2 April 2005

Accepted 16 June 2005 